\title{
Controlled Radical Polymerization of Crude Lignin Bio-oil Containing Multi- hydroxyl Molecules for Methacrylate Polymers and The Potential Applications
}

\author{
Wangda $\mathrm{Qu}^{1}$, Yuerui Huang 2 , Yixin Luo ${ }^{1}$, Sri Kalluru² ${ }^{2}$ Eric Cochran², Michael Forrester ${ }^{2}$, \\ Xianglan Bai ${ }^{*}$ \\ ${ }^{1}$ Department of Mechanical Engineering, Iowa State University, Ames, Iowa, USA, 50011 \\ ${ }^{2}$ Department of Chemical and Biological Engineering, Iowa State University, Ames, Iowa, \\ USA, 50011 \\ * Corresponding author, bx19801@iastate.edu
}

\section{Supporting Information}

Number of pages: 11

Number of table: 1

Number of figures: 8 
Table S1. Datasheet used for quantification of added C $=\mathrm{C}$ for PLMAs

\begin{tabular}{cccccc}
\hline & $\begin{array}{c}\text { Mw } \\
(\mathrm{Da})\end{array}$ & $\begin{array}{c}\text { Mass } \\
(\mathrm{mg})\end{array}$ & $\begin{array}{c}\text { Internal standard } \\
(\mathrm{mg})\end{array}$ & $\begin{array}{c}\text { Peak area } \\
(6-7 \mathrm{ppm})\end{array}$ & $\begin{array}{c}\mathrm{C}=\mathrm{C} \text { in PLMAs } \\
(\mathrm{mol} / \mathrm{mol})\end{array}$ \\
PL* & $600^{*}$ & 24.75 & 4.02 & 5.07 & - \\
PLMA1 & 1046 & 23.5 & 3.99 & 8.88 & 3.58 \\
PLMA2 & 561 & 23.97 & 2.83 & 11.06 & 0.78 \\
& & & & & 1.26 \\
PLMA3 & 803 & 24.12 & 4.05 & 6.51 & \\
\hline
\end{tabular}

*PL after acetylation 
(a)
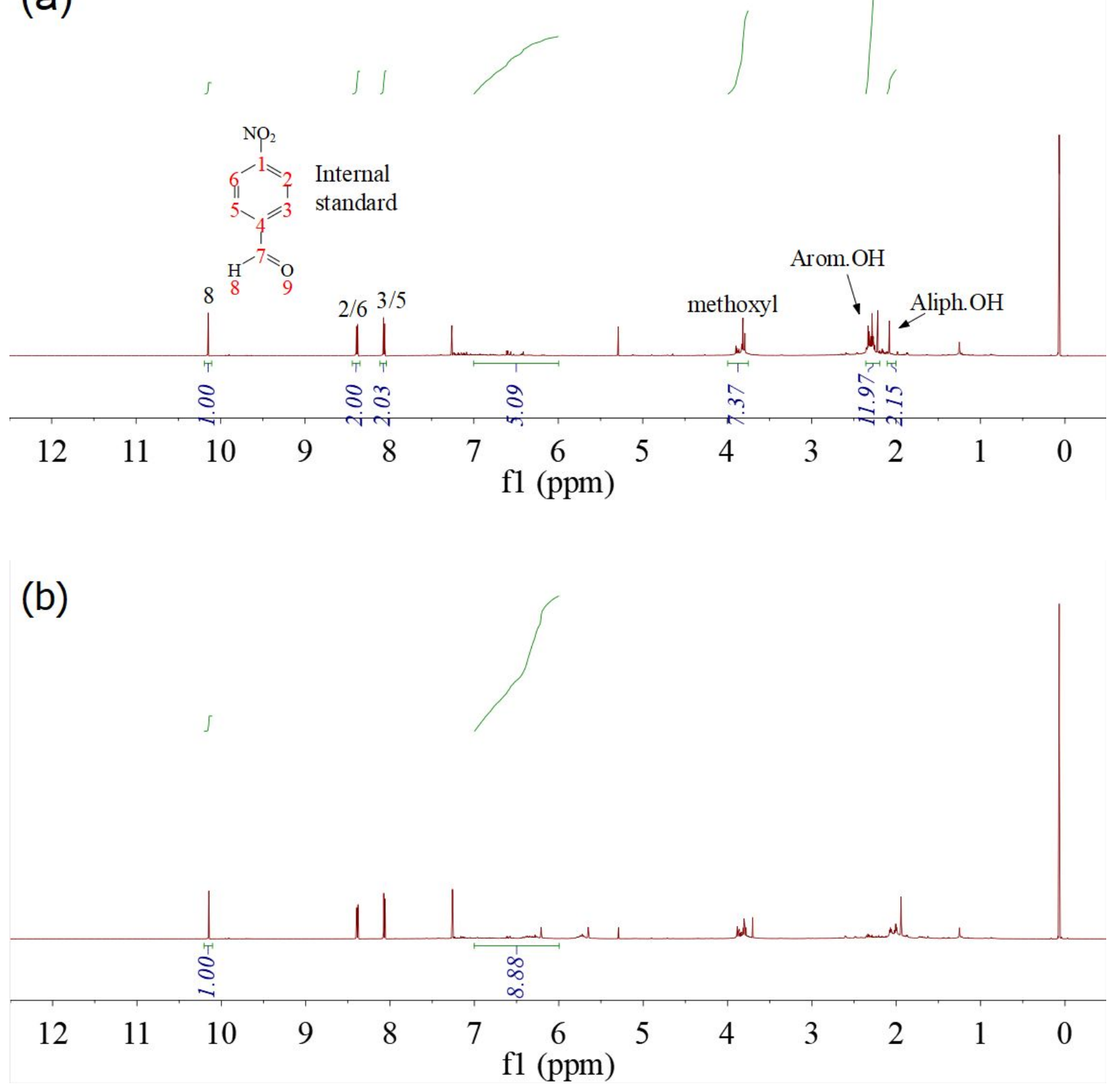

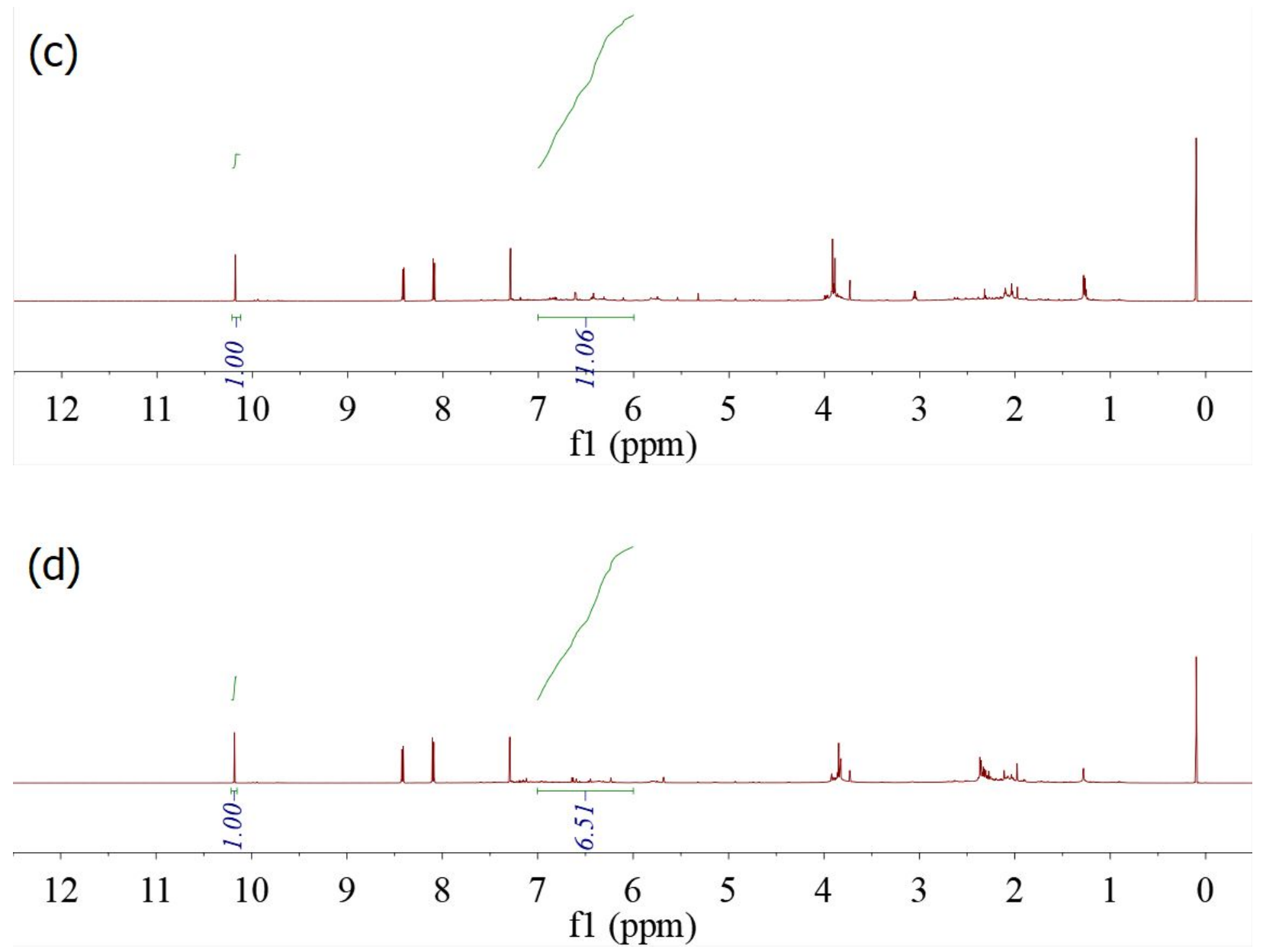

Figure S1. ${ }^{1} \mathrm{H}$ NMR spectra of (a) acetylated PL; (b) PLMA1; (c) PLMA2 and (d) PLMA3. 


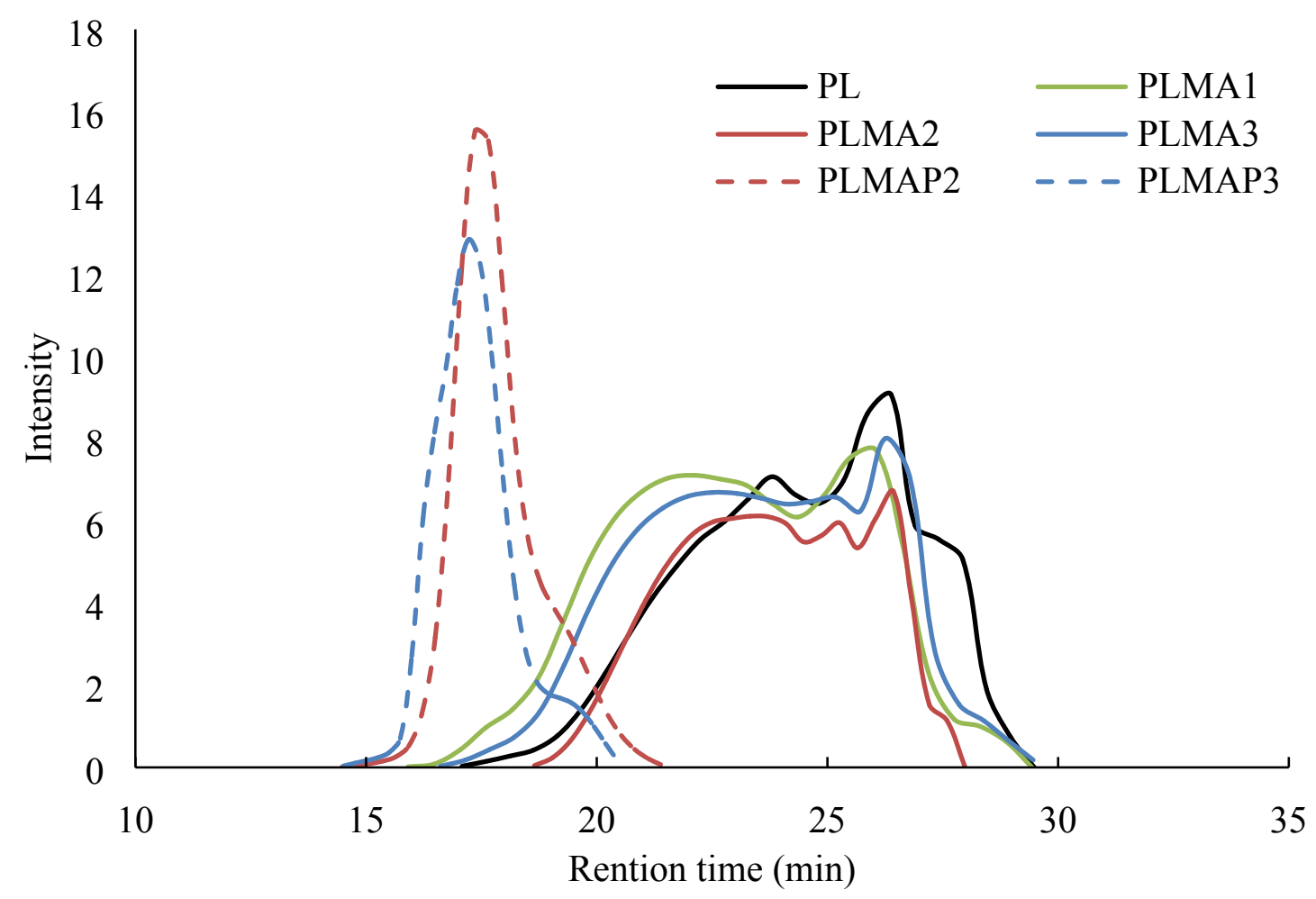

Figure S2. GPC chromatograms of PL, functionized PL (PLMA1, PLMA2, PLMA3) and PL polymers (PLMAP2 and PLMAP3). 


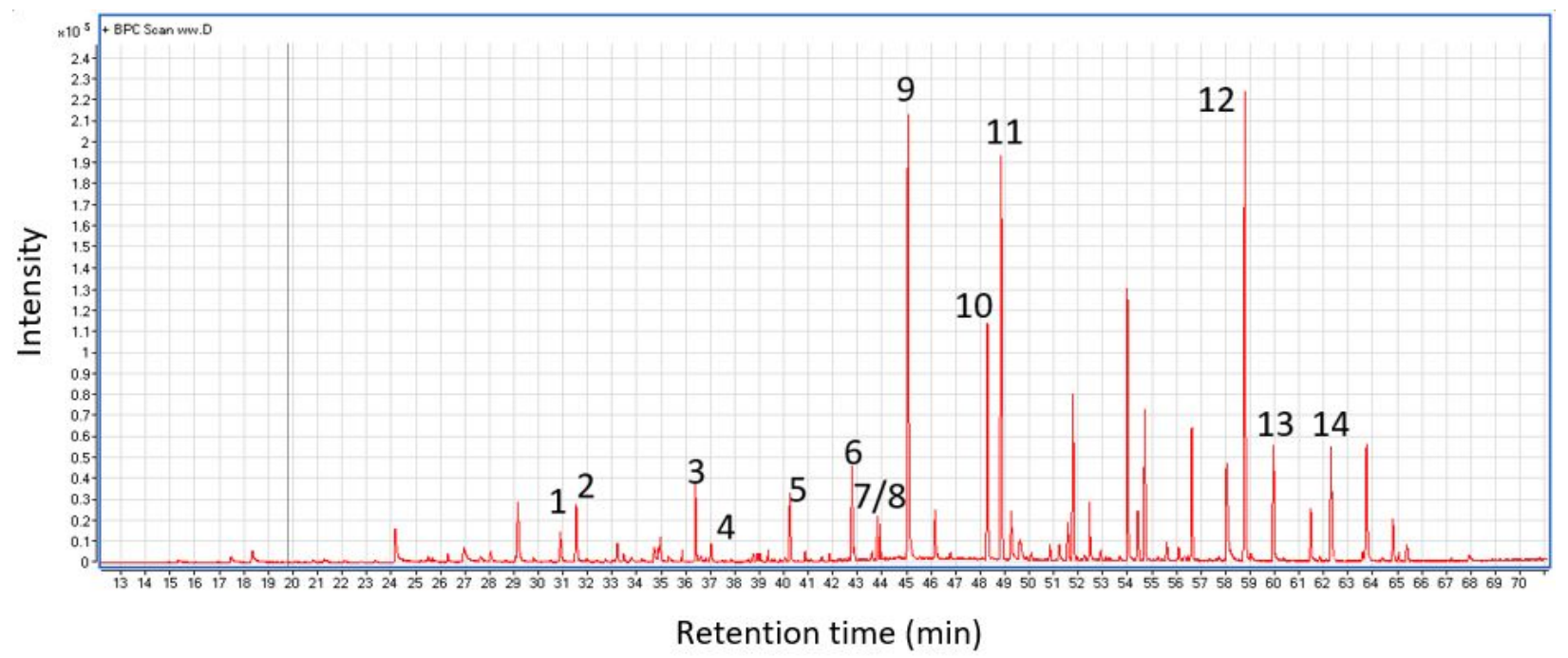

Figure S3. GC/MS detectable phenolic monomers in PL: 1: Phenol; 2: 2-Methoxy phenol; 3: 2Methoxy-5-methylphenol; 4: 3,5-Dimethyl phenol; 5: 3,4-Dimethyl phenol; 6: 2-Methoxy-4vinylphenol; 7: 3-Allyl-6-methoxyphenol; 8: 2-Methoxy-4-propyl phenol; 9: 2,6-Dimethoxy phenol; 10: Trans-isoeugenol; 11: 1,2,4-Trimethoxybenzene; 12: 2,6-Dimethoxy-4-(2-propenyl) phenol; 13: 4-Hydroxy-3,5-dimethoxy benzaldehyde,; 14: 1-(4-Hydroxy-3,5-dimethoxyphenyl) ethanone. 


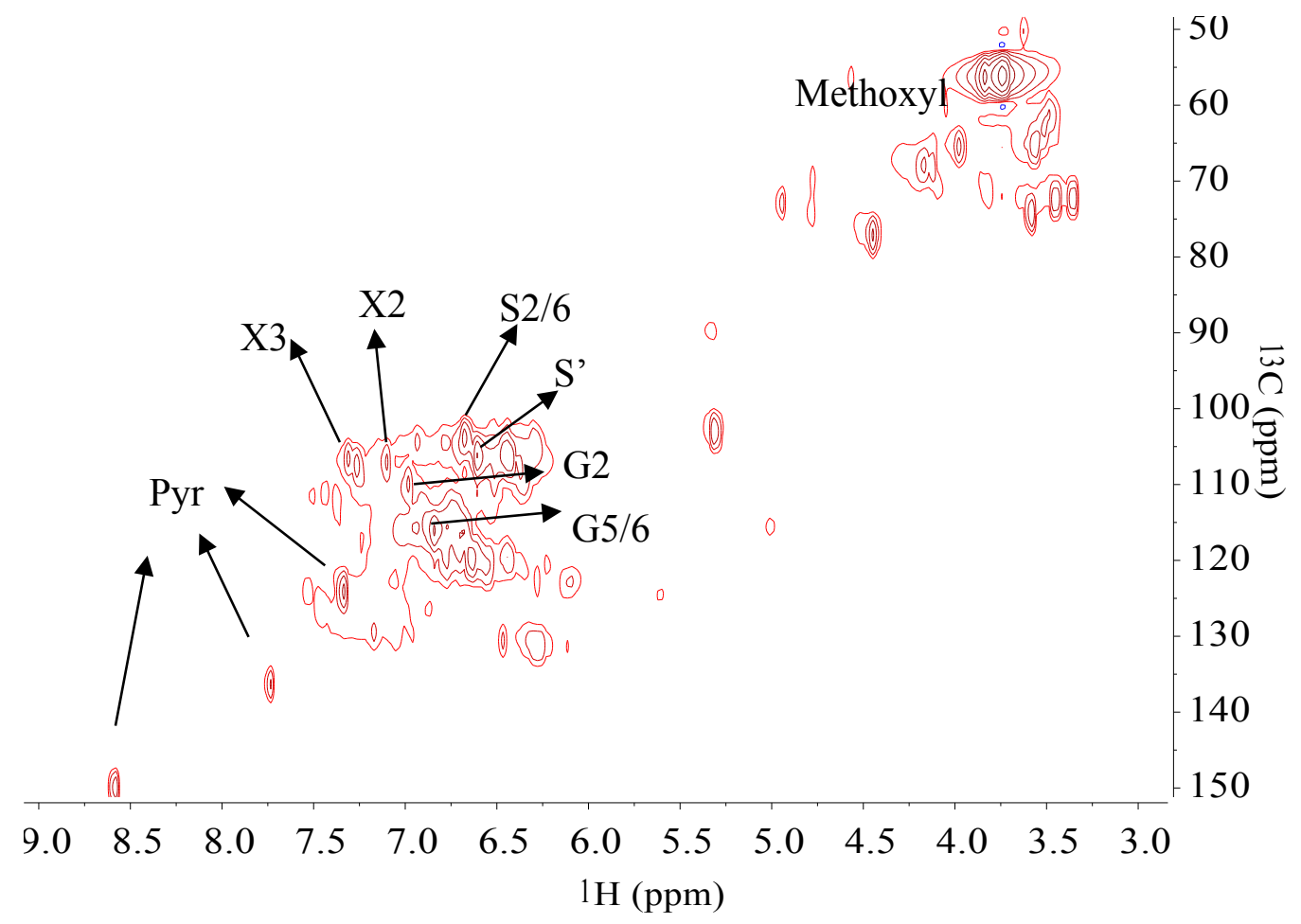

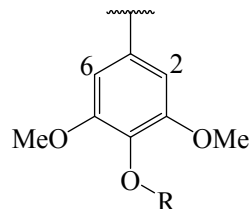

S

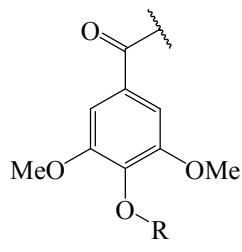

S'

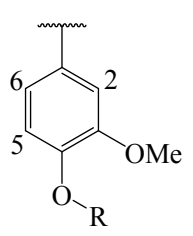

G



$\mathrm{X} 2$

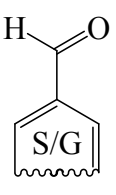

X3

Figure S4. 2D NMR spectrum of PL 



Figure S5. Visual appearances of PL and PLMAPs 


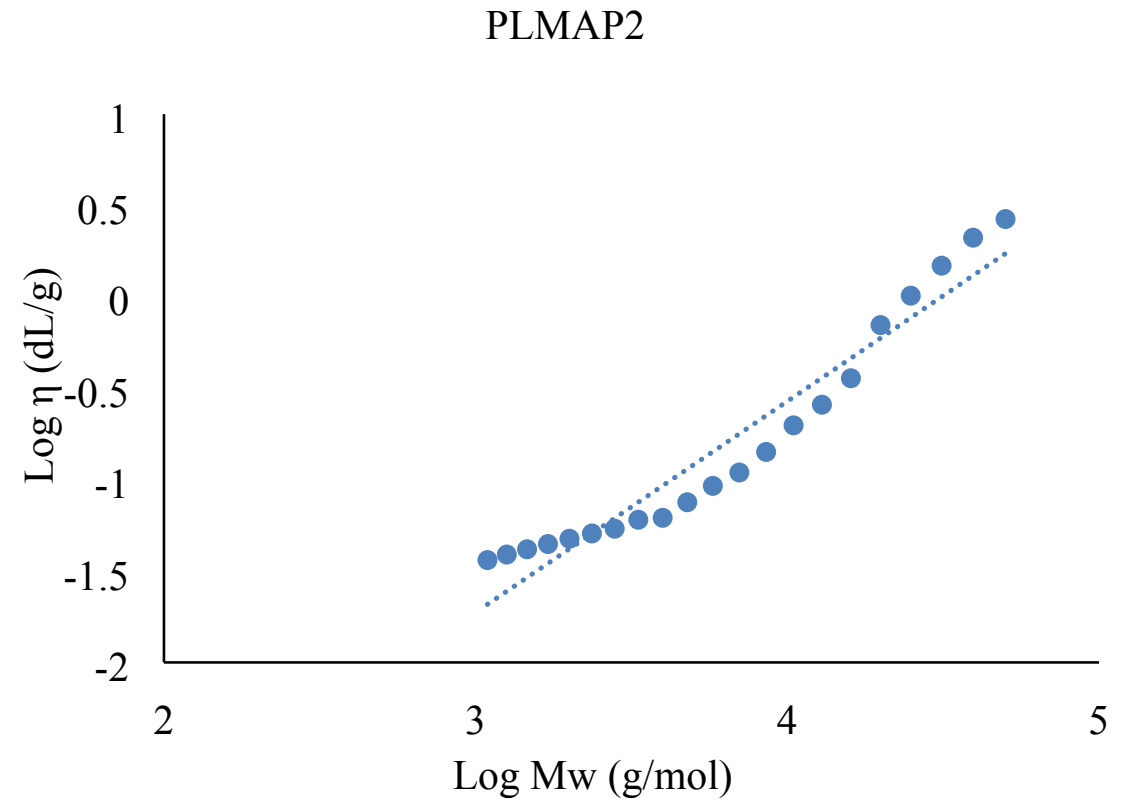

PLMAP3

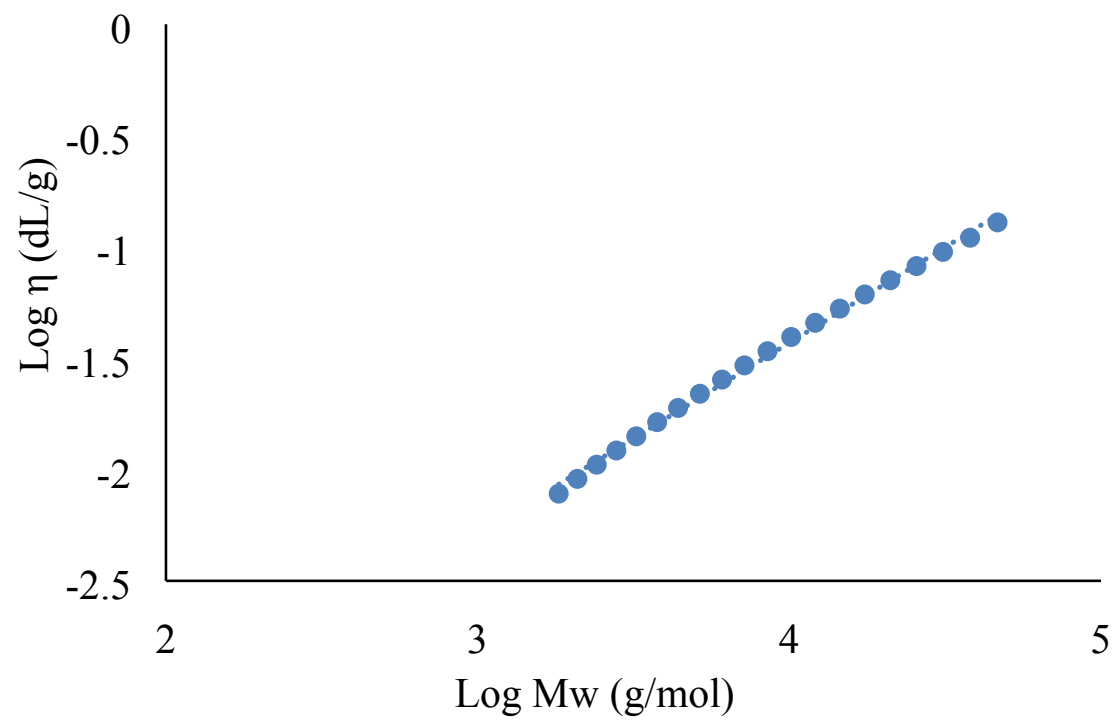

Figure S6. Mark-Houwink plots of PLMAP2 and PLMAP3 


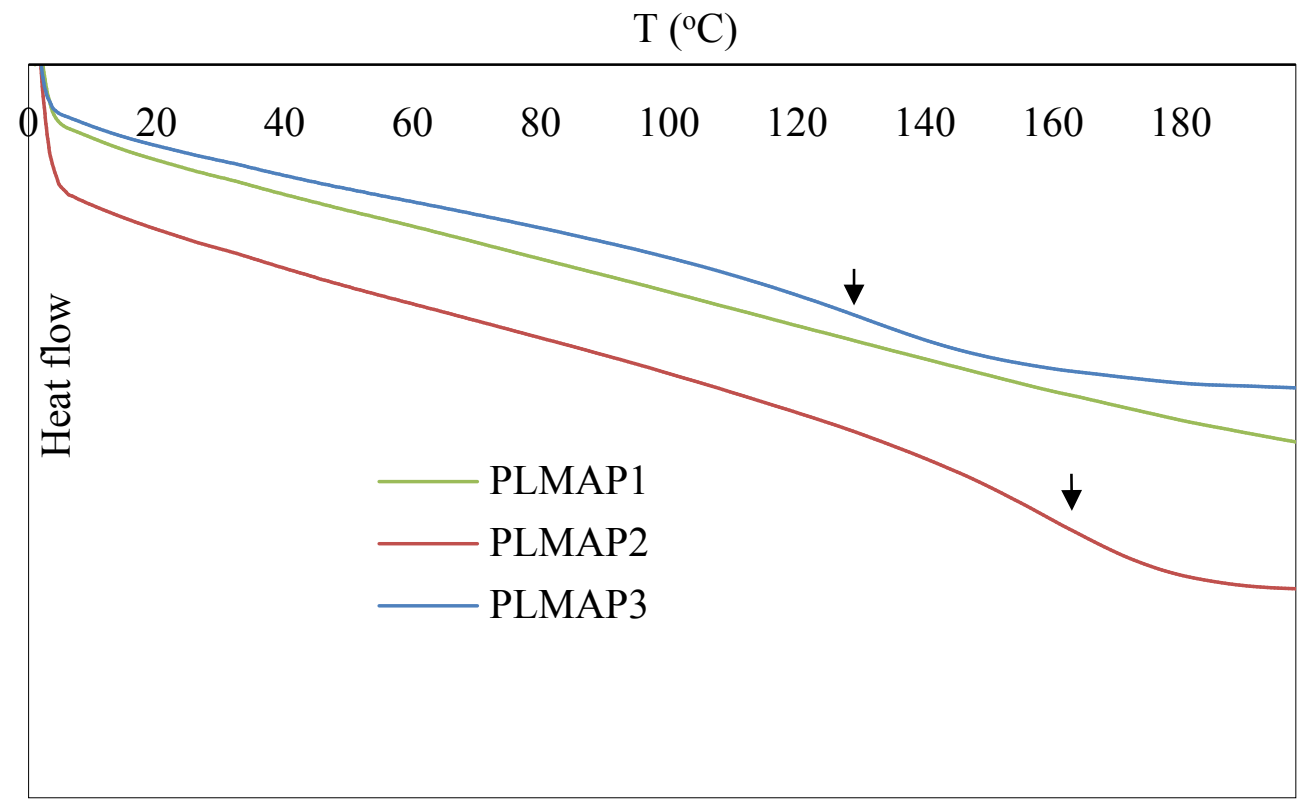

Figure S7. DSC curves of PLMAPs 


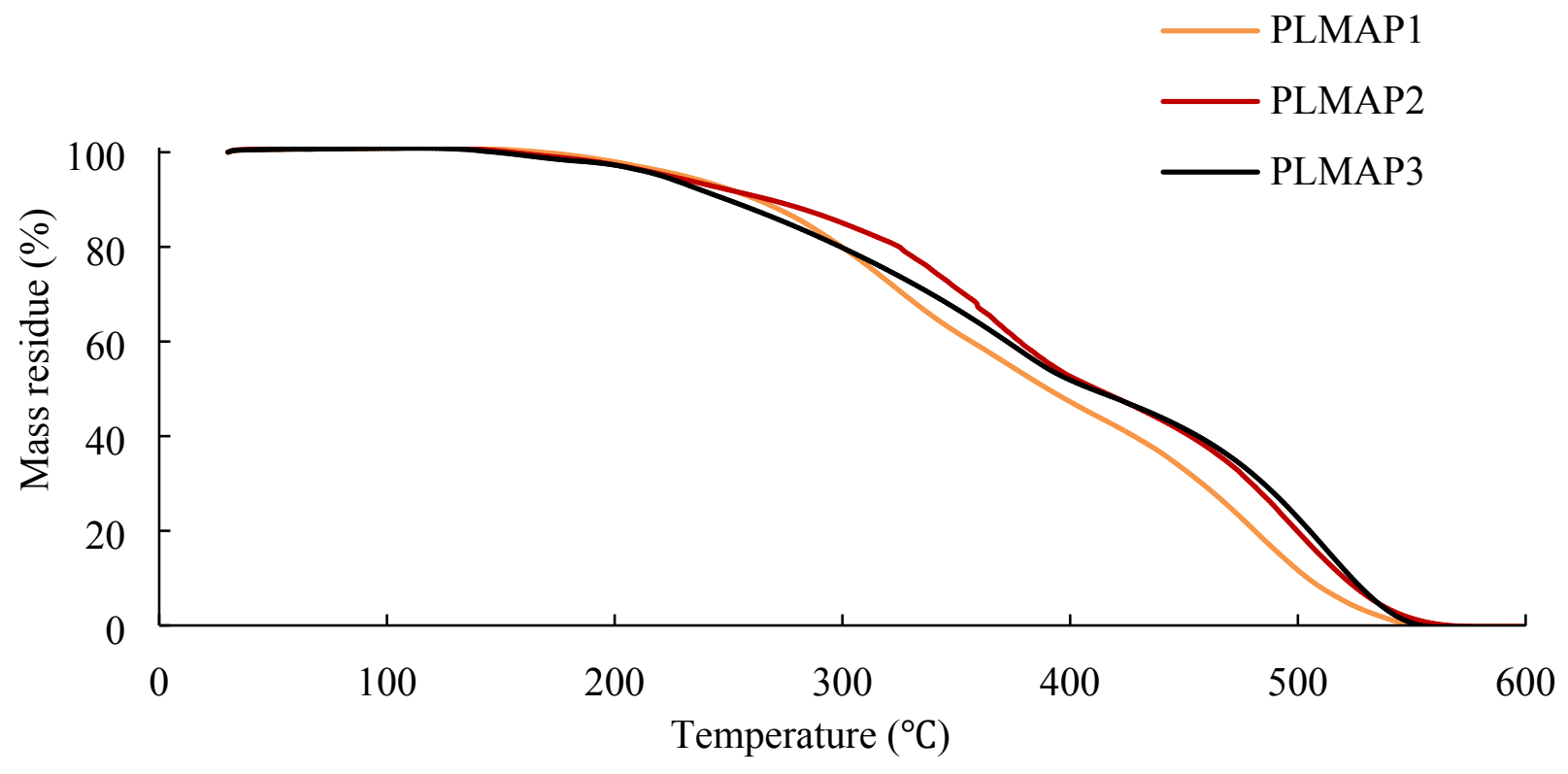

Figure S8. TGA curves of PLMAPs using air 